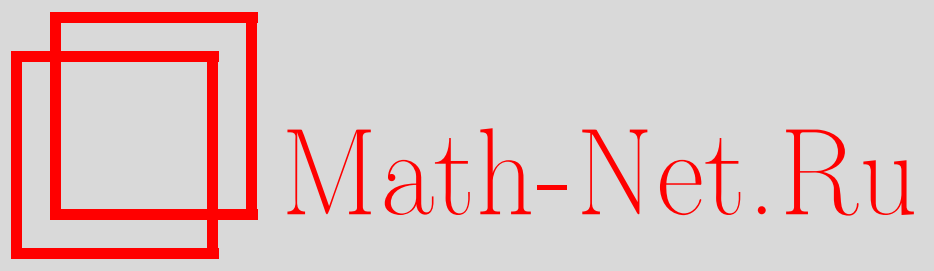

Н. Р. Хуснутдинов, А. Р. Хабибуллин, Поляризация вакуума при наличии нетривиальных граничных условий, ТМФ, 2011, том 166, номер 1, 77-94

DOI: https://doi.org/10.4213/tmf6597

Использование Общероссийского математического портала Math-Net.Ru подразумевает, что вы прочитали и согласны с пользовательским соглашением http://www . mathnet.ru/rus/agreement

Параметры загрузки:

IP: 54.80 .97 .219

26 апреля 2023 г., 14:06:49

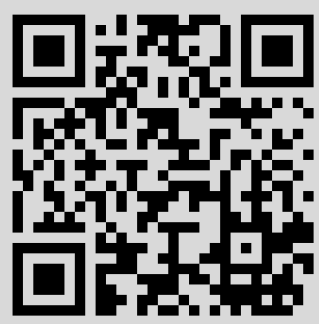




\title{
ФИЗИКА
}

Том 166, № 1

январь, 2011

(C) 2011 г. $\quad$ Н.Р. Хуснутдинов*, А. Р. Хабибуллин ${ }^{\dagger}$

\section{ПОЛЯРИЗАЦИЯ ВАКУУМА ПРИ НАЛИЧИИ НЕТРИВИАЛЬНЫХ ГРАНИЧНЫХ УСЛОВИЙ}

\begin{abstract}
В рамках подхода дзета-регуляризации рассматривается поляризация вакуума скалярного поля при нетривиальных граничных условиях, происхождение которых связано с электродинамикой при наличии проводящей бесконечно тонкой границы. Первый тип граничных условий отвечает случаю, когда поле непрерывно на границе, а его производная испытывает скачок, величина которого пропорциональна полю на границе. При другом типе граничных условий производная поля непрерывна на границе, а скачок, пропорциональный производной поля на границе, испытывает само поле. Получена в явном виде дзета-функция оператора Лапласа скалярного поля при таких граничных условиях и вычислены все коэффициенты теплового ядра. Получено выражение для энергии вакуумных флуктуаций скалярного поля.
\end{abstract}

Ключевые слова: поляризация вакуума, эффект Казимира, дзета-функция, квантовая теория поля.

\section{1. ВВЕДЕНИЕ}

Поляризация вакуума различных полей при наличии границ, называемая эффектом Казимира [1], является макроскопическим проявлением квантовых флуктуаций физических полей. Прецизионные измерения последних лет позволили с высокой точностью измерить этот эффект [2], [3]. Собственно эффектом Казимира называют явление, не зависящее от состава границ, что справедливо на некотором интервале расстояний. По этой причине обоснованным является рассмотрение границы как некоторого граничного условия. Кроме стандартных граничных условий Дирихле, Неймана и Робина в различных задачах возникает множество других нетривиальных граничных условий, отвечающих различным физическим требованиям [2]. Особое место занимают граничные условия, соответствующие наличию потенциалов с точечным носителем (дельтаобразных потенциалов).

Впервые подобные потенциалы были изучены в рамках квантовой механики в работе [4], где было отмечено, что их необходимо рассматривать с большой осторожностью. На пространстве решений уравнения с таким потенциалом гамильтониан

* Казанский (Приволжский) федеральный университет, Казань, Россия. E-mail: 7nail7@gmail.com

† Татарский государственный гуманитарно-педагогический университет, Казань, Россия. E-mail: arphysis@gmail.com 
теряет свойство самосопряженности, и поэтому перестает описывать физически измеряемую величину. В этой ситуации обычно применяется метод самосопряженного расширения операторов (см., например, монографию [5]). Динамика, которая описывается расширенным оператором, не является однозначной, появляется множество динамик, нумеруемых непрерывной или дискретной величиной. Точно решаемые модели квантовой механики с дельтаобразным потенциалом собраны в книге [6].

Впоследствии задачи, содержащие дельтаобразные потенциалы, рассматривались в рамках квантовой теории поля [7]-[18]. С физической точки зрения дельтаобразный потенциал описывает полупрозрачную границу. Математически это выражается в том, что поле не исчезает на границе. При таком подходе границы становятся полупрозрачными. Устремляя параметр $\lambda$, стоящий в потенциале перед дельта-функцией, к бесконечности, мы восстанавливаем граничные условия Дирихле. Основной проблемой, возникающей в таких задачах, является получение конечного выражения для энергии вакуумных флуктуаций [8], [10]-[12], [17]. Впервые это было отмечено в работах [19], [7]. Авторы этих работ указывали на появление расходимости вида $\lambda^{2} / L$ (здесь $L$ - расстояние между пластинами) после перенормировки выражения для энергии вакуумных флуктуаций безмассового скалярного поля. Конечное выражение для энергии Казимира при наличии сингулярного потенциала было получено в работе [8].

В серии работ [20]-[24] утверждается, что невозможно описать эффект Казимира в рамках квантовой электродинамики. Показано, что после проведения всех перенормировок результат расходится в пределе Дирихле $\lambda \rightarrow \infty$. На основании этого авторы работ [20]-[24] сделали заключение о том, что эффект Казимира не является следствием вакуумных колебаний электромагнитного поля. Подобные трудности также отмечены в работе [14], где рассматривалось электромагнитное поле при наличии граничных условий для поперечно-электрической (ТЕ) и поперечно-магнитной (ТМ) поляризаций и было показано, что энергия Казимира логарифмически расходится. Возможное решение этой проблемы состоит в корректном проведении перенормировок [25].

Целью настоящей работы является детальное исследование эффекта Казимира для скалярного поля, как массивного $(m \neq 0)$, так и безмассового $(m=0)$, при наличии граничных условий, возникающих для ТЕ-и ТМ-поляризаций. Основным требованием к перенормировке является то, что полученное выражение для энергии должно удовлетворять всем необходимым предельным случаям:

1) в пределе $\lambda \rightarrow \infty$ в месте нахождения дельтаобразного потенциала восстанавливаются граничные условия Дирихле (или Неймана), и ситуация сводится к уже известной с каким-либо набором идеально проводящих металлических пластин;

2 ) в пределе $m \rightarrow \infty$ энергия нулевых колебаний стремится к нулю.

Мы рассматриваем различные виды перенормировок и показываем, что корректным является вычитание слагаемых, сохраняющихся, когда границы удаляются на бесконечность. Для безмассового поля это вычитание эквивалентно вычитанию слагаемых, сохраняющихся в пределе $\lambda \rightarrow \infty$.

Статья состоит из шести частей. Раздел 2 посвящен краткому обзору подхода, основанного на дзета-регуляризации, к вычислению энергии вакуумных флуктуаций. В разделе 3 рассматриваются граничные условия для ТЕ- и ТМ-поляризаций и их применение к скалярному полю; приводятся дзета-функции безмассового и 
массивного скалярных полей при наличии данных граничных условий. В разделе 4 вычисляется энергия вакуумных флуктуаций скалярного поля, безмассового (п. 4.1) и массивного (п. 4.2); рассмотрены различные перенормировочные условия. Обсуждению результатов посвящен раздел 5. В приложении приводятся формулы для коэффициентов теплового ядра $a_{k}$ и $b_{k}$.

\section{2. ЭНЕРГИЯ КАЗИМИРА В ПОДХОДЕ ДЗЕТА-РЕГУЛЯРИЗАЦИИ}

В квантовой теории поля метод обобщенной дзета-функции впервые был предложен в работах [26] и [27] (см. также обзор [28]). Применительно к эффекту Казимира этот метод был развит в работах [29]-[32]. Отметим кратко основные моменты метода дзета-регуляризации, важные при вычислении энергии нулевых колебаний. Энергия $E$ нулевых колебаний поля определяется как полусумма (интеграл для непрерывной переменной) по всему спектру энергии собственных значений $\omega_{(n)}=\sqrt{\lambda_{(n)}^{2}+m^{2}}$ оператора лапласовского типа с какими-либо граничными условиями,

$$
E=\frac{1}{2} \sum_{(n)} \sqrt{\lambda_{(n)}^{2}+m^{2}}
$$

Здесь параметр $m$ является массой поля. Определенная таким образом величина $E$ расходится. В рамках подхода дзета-регуляризации это выражение рассматривают как аналитическое продолжение в точку $s=0$ функции

$$
E(s)=\frac{1}{2} \mu^{2 s} \zeta\left(s-\frac{1}{2}, D_{N}\right)
$$

где

$$
\zeta\left(s, D_{N}\right)=\sum_{(n), j}\left(\lambda_{(n), j}^{2}+m^{2}\right)^{-s}
$$

является обобщенной дзета-функцией оператора $D_{N}=-\triangle_{N}+m^{2}+V$. Параметр $\mu$ с размерностью массы введен для того, чтобы величина $E(s)$ имела размерность энергии. Здесь мультииндекс $(n)$ обозначает дискретные числа задачи, а индекс $j$ нумерует соответствующие решения граничной задачи. Регуляризованная энергия $E(s)$ является аналитической функцией от параметра $s$, ряд в (2) сходится для $s>1$. Чтобы получить энергию нулевых колебаний, необходимо вычислить предел $s \rightarrow 0$ в выражении для энергии (2) и соответственно предел $s \rightarrow 1 / 2$ в выражении для дзета-функции (3). В этом пределе функция $E(s)$ расходится, и расходимости можно выделить в явном как полюсы гамма-функций, используя асимптотическое разложение дзета-функции

$$
\zeta_{\text {as }}\left(s, D_{N}\right)=\frac{1}{(4 \pi)^{N / 2}} \sum_{n=0}^{\infty} b_{n / 2} m^{-2 s-n+N} \frac{\Gamma(s+(n-N) / 2)}{\Gamma(s)},
$$

где $N$ - размерность пространства и $b_{n / 2}$ - коэффициенты теплового ядра, впервые исследованные в работах [33], [34]. Простейшим и удобным способом перенормировки является следующий [31]. Для выделения расходящихся слагаемых вычисляем предел больших масс $m \rightarrow \infty$ и сохраняем все слагаемые, которые выживают в этом пределе, $E^{\operatorname{div}}(s)=\lim _{m \rightarrow \infty} E(s)$. Очевидно, что эти слагаемые необходимо удалить, 
поскольку в этом классическом пределе энергия должна равняться нулю. Такое вычитание можно интерпретировать как перенормировку параметров классической части энергии [31]. Тогда перенормированное значение энергии нулевых колебаний есть $E^{\mathrm{ren}}=\lim _{s \rightarrow 0}\left(E(s)-E^{\operatorname{div}}(s)\right)$, где расходящаяся часть имеет следующий вид:

$$
E^{\operatorname{div}}(s)=\left(\frac{\mu}{m}\right)^{2 s} \frac{1}{2(4 \pi)^{N / 2}} \sum_{n=0}^{N+1} b_{n / 2} m^{N+1-n} \frac{\Gamma(s+(n-N-1) / 2)}{\Gamma(s-1 / 2)} .
$$

В большинстве случаев найти спектр оператора $D_{N}$ в явном виде и просуммировать ряд невозможно. Для получения конечного выражения воспользуемся следующим приемом. Спектр энергии получается в результате решения некоторого функционального соотношения

$$
\Psi\left(\lambda_{(n), j}, R\right)=0
$$

где параметр $R$ характеризует граничные условия модели, а $\lambda_{(n), j}=\sqrt{\omega_{(n)}^{2}-m^{2}}-$ кинетическую энергию. В рамках подхода, развитого в работе [31], суммирование по $j$ можно конвертировать в контурный интеграл и затем деформировать контур на мнимую ось. В итоге получается следующее выражение для регуляризованной энергии вакуумных флуктуаций:

$$
E^{\mathrm{reg}}(s)=-\mu^{2 s} \frac{\cos (\pi s)}{2 \pi} \sum_{(n)} \int_{m}^{\infty} d k\left(k^{2}-m^{2}\right)^{-s+1 / 2} \partial_{k} \ln \Psi_{(n)}(i k, R) .
$$

Представим выражение для перенормированной энергии как $E^{\mathrm{ren}}=E^{\mathrm{num}}+E^{\mathrm{an}}$, где

$$
\begin{aligned}
E^{\mathrm{num}} & =-\frac{1}{2 \pi} \sum_{(n)} \int_{m}^{\infty} d k \sqrt{k^{2}-m^{2}}\left(\partial_{k} \ln \Psi_{(n)}(i k, R)-\partial_{k} \ln \Psi_{(n)}^{\mathrm{un}}(i k, R)\right), \\
E^{\mathrm{an}} & =\lim _{s \rightarrow 0}\left(E^{\mathrm{un}}(s)-E^{\operatorname{div}}(s)\right), \\
E^{\mathrm{un}}(s) & =-\mu^{2 s} \frac{\cos (\pi s)}{2 \pi} \sum_{(n)} \int_{m}^{\infty} d k\left(k^{2}-m^{2}\right)^{-s+1 / 2} \partial_{k} \ln \Psi_{(n)}^{\mathrm{un}}(i k, R),
\end{aligned}
$$

a $\Psi_{(n)}^{\text {un }}(i k, R)$ представляет собой равномерно сходящееся асимптотическое разложение функции $\Psi_{(n)}(i k, R)$. Здесь $E^{\text {num }}-$ часть энергии, рассчитываемая численно, $E^{\mathrm{an}}$ является аналитической конечной частью энергии, в которой происходит вся процедура перенормировки.

\section{3. ГРАНИЧНЫЕ УСЛОВИЯ}

Рассмотрим кратко происхождение граничных условий, используемых в настоящей работе. В сферически-симметричном случае электромагнитное поле имеет две независимые поляризации, которые в литературе принято называть ТЕ- и ТМ-поляризациями. Электромагнитное поле представляется в следующем виде $(k=\omega / c)$ :

$$
\begin{aligned}
& \mathbf{E}=\sum_{l, m} \int_{0}^{\infty} \frac{d k}{\pi \sqrt{2 \omega}}\left\{e^{-i \omega t} \mathbf{E}_{l m}+e^{i \omega t} \mathbf{E}_{l m}^{*}\right\}, \\
& \mathbf{B}=\sum_{l, m} \int_{0}^{\infty} \frac{d k}{\pi \sqrt{2 \omega}}\left\{e^{-i \omega t} \mathbf{B}_{l m}+e^{i \omega t} \mathbf{B}_{l m}^{*}\right\},
\end{aligned}
$$


где для ТЕ-поляризации

$$
\mathbf{E}_{l m}^{\mathrm{TE}}=\frac{1}{r} f(k r) \mathbf{L} Y_{l m}(\Omega), \quad \mathbf{B}_{l m}^{\mathrm{TE}}=-\frac{i}{k} \nabla \times \mathbf{E}_{l m}^{\mathrm{TE}},
$$

а для ТМ-поляризации

$$
\mathbf{B}_{l m}^{\mathrm{TM}}=\frac{1}{r} g(k r) \mathbf{L} Y_{l m}(\Omega), \quad \mathbf{E}_{l m}^{\mathrm{TM}}=\frac{i}{k} \nabla \times \mathbf{B}_{l m}^{\mathrm{TM}} .
$$

Здесь $\mathbf{L}$ - оператор углового момента. В стандартном орторепере $\left(e_{r}, e_{\theta}, e_{\varphi}\right)$ получаем в явном виде

$$
\begin{aligned}
r \mathbf{E}_{l m}^{\mathrm{TE}} & =\left(0, \frac{i f}{\sin \theta} \partial_{\varphi} Y_{l m},-i f \partial_{\theta} Y_{l m}\right), \\
r \mathbf{B}_{l m}^{\mathrm{TM}} & =\left(0, \frac{i g}{\sin \theta} \partial_{\varphi} Y_{l m},-i g \partial_{\theta} Y_{l m}\right),
\end{aligned}
$$

что оправдывает название поля как поперечно-электрического и поперечно-магнитного. Наличие бесконечно тонкой проводящей сферы радиуса $R$ с поверхностной плотностью заряда $n$ приводит к следующим уравнения на скачок радиальной части полей: для ТЕ-моды

$$
f(R+0)-f(R-0)=0, \quad f^{\prime}(R+0)-f^{\prime}(R-0)=\Omega f(R),
$$

для ТМ-моды

$$
g^{\prime}(R+0)-g^{\prime}(R-0)=0, \quad g(R+0)-g(R-0)=-\frac{\Omega}{k^{2}} g^{\prime}(R),
$$

где $\Omega=4 \pi n e^{2} / m c^{2}$. Аналогичные условия при наличии плоской границы получены в работе [15], а также в недавней работе [35] при описании взаимодействия идеального проводника с графеном в модели Дирака. Интересная интерпретация написанных выше граничных условий как матричных уравнений с оператором, зависящим от частоты, приведена в статье [36]. В пределе $\Omega \rightarrow \infty$ граничные условия для ТЕи ТМ-мод переходят соответственно в граничные условия Дирихле и Неймана.

Рассмотрим действительное скалярное поле $\phi(x)$ в $(3+1)$-мерном пространстве Минковского при наличии двух идеально проводящих пластин в точках $x= \pm L$ с граничными условиями Дирихле или Неймана на них, а также источника с сингулярным потенциалом в точке $x=0$, расположенного между этими пластинами. Скалярное поле подчиняется стандартному уравнению

$$
\left(\frac{\partial^{2}}{\partial t^{2}}-\triangle+m^{2}\right) \phi(x)=0 .
$$

Сингулярный потенциал, имеющий точечный носитель, описывается граничными условиями в точке $x=0$. Мы рассмотрим специфические условия, которые обсуждались в работах [20], [14], [25] в контексте ТЕ- и ТМ-поляризаций электромагнитного поля.

Уравнение на собственные значения оператора $D_{N}$ выглядит как $\phi^{\prime \prime}=-k^{2} \phi$ и имеет общее решение, содержащее четыре константы:

$$
\phi= \begin{cases}A^{-} e^{i k x}+B^{-} e^{-i k x}, & x<0, \\ A^{+} e^{i k x}+B^{+} e^{-i k x}, & x>0 .\end{cases}
$$


Мы рассматриваем три вида граничных условий (6): граничные условия первого рода (ТЕ-мода)

$$
\phi(+0)-\phi(-0)=0, \quad \phi^{\prime}(+0)-\phi^{\prime}(-0)=\lambda \phi(0), \quad \phi( \pm L)=0 ;
$$

граничные условия второго рода при наличии границ Дирихле (ТМ1-мода)

$$
\phi^{\prime}(+0)-\phi^{\prime}(-0)=0, \quad \phi(+0)-\phi(-0)=-\frac{\lambda}{k^{2}} \phi^{\prime}(0), \quad \phi( \pm L)=0 ;
$$

граничные условия второго рода при наличии границ Неймана (ТМ2-мода)

$$
\phi^{\prime}(+0)-\phi^{\prime}(-0)=0, \quad \phi(+0)-\phi(-0)=-\frac{\lambda}{k^{2}} \phi^{\prime}(0), \quad \phi^{\prime}( \pm L)=0 .
$$

Отметим, что в случае ТЕ-поляризации можно изначально рассматривать уравнение движения поля, содержащее сингулярный потенциал,

$$
\left(\frac{\partial^{2}}{\partial t^{2}}-\triangle+m^{2}+V\right) \phi(x)=0
$$

где $V=\lambda \delta(x)$. Тогда граничные условия для ТЕ-поляризации будут фактически следствием данного уравнения. Простой интерпретации граничных условий для ТМ-моды в терминах сингулярного потенциала нет. При $\lambda \rightarrow \infty$ восстанавливаются граничные условия Дирихле и Неймана для ТЕ- и ТМ-мод соответственно. Поэтому в пределе $\lambda \rightarrow \infty$ мы получаем три пластины с граничными условиями Дирихле в случае ТЕ-моды и две пластины с условиями Дирихле, между которыми располагается пластина с условием Неймана на ней, в случае ТМ-моды. Расстояние между всеми пластинами равно $L$.

Для нахождения энергии нулевых колебаний в рамках дзета-подхода из граничных условий (12), которые представляют собой однородные системы линейных уравнений на четыре неизвестные константы $A^{ \pm}, B^{ \pm}$, получим функции (на мнимой оси), приравнивание которых к нулю определяет спектр, для трех указанных случаев:

$$
\begin{aligned}
\Psi_{\mathrm{TE}}(i k) & =4 \operatorname{sh}(k L)\{2 k \operatorname{ch}(k L)+\lambda \operatorname{sh}(k L)\}, \\
\Psi_{\mathrm{TM} 1}(i k) & =4 \operatorname{ch}(k L)\{2 k \operatorname{sh}(k L)+\lambda \operatorname{ch}(k L)\}, \\
\Psi_{\mathrm{TM} 2}(i k) & =\Psi_{\mathrm{TE}}(i k) .
\end{aligned}
$$

При решении нашей задачи будет полезным следующее соотношение, понижающее размерность оператора для дзета-функции:

$$
\zeta\left(s, D_{3}\right)=\frac{\zeta\left(s-1, D_{1}\right)}{4 \pi(s-1)},
$$

которое получается простым интегрированием функции (3) по непрерывному спектру в плоскости пластин. Вычисления существенно упрощаются, и мы имеем дело с дзета-функцией для одномерного оператора:

$$
\zeta\left(s, D_{1}\right)=\frac{\sin (\pi s)}{\pi} \int_{m}^{\infty} d k\left(k^{2}-m^{2}\right)^{-s} \partial_{k} \ln \Psi(i k) .
$$


Определим функцию, зависящую от параметра $\lambda$, следующим соотношением:

$$
\zeta_{\lambda}\left(s, D_{1}\right)=\zeta\left(s, D_{1}\right)-\left.\zeta\left(s, D_{1}\right)\right|_{\lambda=0}=\frac{\sin (\pi s)}{\pi} \int_{m}^{\infty} d k\left(k^{2}-m^{2}\right)^{-s} \partial_{k} \ln \Psi^{\lambda}(i k),
$$

где $\left.\zeta\left(s, D_{1}\right)\right|_{\lambda=0}$ определяет стандартную энергию Казимира для двух пластин, отстоящих друг от друга на расстояние $2 L$, с граничными условиями Дирихле или Неймана без сингулярного потенциала. Согласно такому определению функции (14) примут вид

$$
\begin{aligned}
\Psi_{\mathrm{TE}}^{\lambda}(i k) & =1+\frac{\lambda}{2 k} \operatorname{th}(k L), \\
\Psi_{\mathrm{TM} 1}^{\lambda}(i k) & =1+\frac{\lambda}{2 k} \operatorname{cth}(k L), \\
\Psi_{\mathrm{TM} 2}^{\lambda}(i k) & =\Psi_{\mathrm{TE}}^{\lambda}(i k) .
\end{aligned}
$$

Видно, что спектральные функции для ТЕ- и ТМ2-мод совпадают. Таким образом, достаточно рассмотреть задачу для ТЕ- и ТМ1-поляризаций.

Рассмотрим случай удаленных на бесконечность пластин $(L \rightarrow \infty)$. Очевидно, что энергия Казимира в пустом пространстве с уединенной границей равна нулю. Функция $\Psi^{\lambda}(i k)$ в пределе $L \rightarrow \infty$ одинакова для всех трех случаев $(17)$ :

$$
\Psi^{\lambda}(i k)=1+\frac{\lambda}{2 k}
$$

Соответствующая ей дзета-функция равна

$$
\zeta_{\lambda}\left(s, D_{1}\right)=-\frac{\sin (\pi s)}{\pi} \int_{m}^{\infty} d k\left(k^{2}-m^{2}\right)^{-s} \frac{\lambda}{k(\lambda+2 k)} .
$$

Дзета-функция для массивного поля $(m \neq 0)$ выражается формулой

$$
\begin{aligned}
& \zeta_{\lambda}\left(s, D_{1}\right)=-\frac{1}{2} m^{-2 s}\left\{1-\left(1-z^{2}\right)^{-s}-\frac{\Gamma(1-s) \Gamma(s+1 / 2)}{\pi^{3 / 2} s z} \sin (\pi s) \times\right. \\
& \left.\quad \times\left[\left(1-z^{2}\right)_{2} F_{1}\left(1, s+\frac{1}{2} ;-\frac{1}{2} ; z^{2}\right)+\left((2 s+3) z^{2}-1\right){ }_{2} F_{1}\left(1, s+\frac{1}{2} ; \frac{1}{2} ; z^{2}\right)\right]\right\},
\end{aligned}
$$

где $z=\lambda / 2 m$ и ${ }_{2} F_{1}(\cdot)$ - гипергеометрическая функция. Используя разложение выражения (19) по степеням $1 / k$, несложно получить все коэффициенты теплового ядра, которые приведены в приложении.

В безмассовом случае $m=0$ мы имеем

$$
\zeta_{\lambda}\left(s, D_{1}\right)=\frac{2^{2 s-1}}{\lambda^{2 s} \cos (\pi s)}, \quad \zeta_{\lambda}\left(s, D_{3}\right)=\frac{2^{2 s-5}}{\pi(1-s) \lambda^{2 s-2} \cos (\pi s)} .
$$

Эти соотношения совпадают с выражениями для дзета-функции в случае ТЕ-моды, полученными в работе [15]. В этом случае можно вычислить все коэффициенты теплового ядра, используя разложение выражения (19) по степеням $z=1 /(k+\lambda / 2)$ вместо разложения по степеням $z=1 / k$ (см. приложение). 
Перенормировка, как хорошо известно, сводится к переопределению параметров классической части энергии. В нашей задаче классическая часть энергии неизвестна, поэтому в качестве нормировочного условия мы используем требование, чтобы в пределе $\lambda \rightarrow \infty$ энергия соответствовала наличию трех (или одной в случае ТЕ-моды) пластин с граничными условиями Дирихле на каждой из них. Это требование является естественным, поскольку, как отмечалось выше, в пределе $\lambda \rightarrow \infty$ восстанавливаются условия Дирихле. Такое условие дает возможность подобрать значение параметра $\mu$ так, чтобы выполнялось условие равенства нулю энергии Казимира для одной пластины. Таким образом, после перенормировки мы получим выражение для энергии Казимира, удовлетворительно описывающее поведение энергии для произвольного $\lambda$.

\section{4. ЭНЕРГИЯ КАЗИМИРА СКАЛЯРНОГО ПОЛЯ ПРИ НАЛИЧИИ НЕТРИВИАЛЬНЫХ ГРАНИЧНЫХ УСЛОВИЙ}

Получим вначале выражения для стандартной энергии Казимира $E_{\text {Cas }}$ в рамках подхода дзета-регуляризации. Рассмотрим две пластины, отстоящие на расстояние $L$ друг от друга, без сингулярных источников. В первом случае мы имеем две пластины с условиями Дирихле на них (DD-случай), во втором случае на одной пластине выполнено условие Дирихле, а на другой - условие Неймана (DN-случай). Спектры энергии получаются приравниванием нулю функций

$$
\Psi_{\mathrm{DD}}(k)=\frac{\sin (k L)}{k}, \quad \Psi_{\mathrm{DN}}(k)=\cos (k L) .
$$

Тогда выражения для энергии Казимира $E_{\text {Cas }}$ имеют следующий вид: в безмассовом случае

$$
\begin{aligned}
& E_{\mathrm{Cas}}^{\mathrm{DD}}=-\frac{L}{6 \pi^{2}} \int_{0}^{\infty} d k \frac{k^{3}}{e^{2 k L}-1}=-\frac{\pi^{2}}{1440 L^{3}}, \\
& E_{\mathrm{Cas}}^{\mathrm{DN}}=\frac{L}{6 \pi^{2}} \int_{0}^{\infty} d k \frac{k^{3}}{e^{2 k L}+1}=\frac{7}{8} \frac{\pi^{2}}{1440 L^{3}}=-\frac{7}{8} E_{\mathrm{Cas}}^{\mathrm{DD}},
\end{aligned}
$$

а для массивного поля $(\beta=m L)$

$$
\begin{aligned}
& E_{\mathrm{Cas}}^{\mathrm{DD}}=-\frac{m^{3} \beta}{6 \pi^{2}} \int_{1}^{\infty} d x \frac{\left(x^{2}-1\right)^{3 / 2}}{e^{2 x \beta}-1}=-\frac{1}{6 \pi^{2} L^{3}} \int_{\beta}^{\infty} d k \frac{\left(k^{2}-\beta^{2}\right)^{3 / 2}}{e^{2 k}-1}, \\
& E_{\mathrm{Cas}}^{\mathrm{DN}}=\frac{m^{3} \beta}{6 \pi^{2}} \int_{1}^{\infty} d x \frac{\left(x^{2}-1\right)^{3 / 2}}{e^{2 x \beta}+1}=\frac{1}{6 \pi^{2} L^{3}} \int_{\beta}^{\infty} d k \frac{\left(k^{2}-\beta^{2}\right)^{3 / 2}}{e^{2 k}+1}
\end{aligned}
$$

4.1. Безмассовое скалярное поле. Для вычисления энергии вакуумных флуктуаций (энергии Казимира) скалярного поля с нулевой массой в случае пластин, расположенных на расстоянии $L$ друг от друга, мы также будем использовать нормировочное условие, выражающееся в том, что при определенных предельных значениях параметра $\lambda$ энергия нулевых колебаний для различных граничных условий будет равна стандартной энергии Казимира для двух или трех пластин в данном поле. 
Рассмотрим безмассовое скалярное поле при наличии сингулярного источника, удаленного на одинаковое расстояние $L$ от пластин, с различными типами граничных условий. Одной из основных задач является нахождение равномерно сходящегося асимптотического разложения функции $\Psi(i k)$ для больших $k$. Асимптотика функции $\Psi(i k)$ при $k \rightarrow \infty$ для всех типов граничных условий будет иметь вид

$$
\left(\Psi^{\lambda}\right)^{\text {as }}=1+\frac{\lambda}{2 k}+O\left(e^{-k L}\right) .
$$

Полученное разложение совпадает с разложением в случае границ, удаленных на бесконечность $(L \rightarrow \infty)$. Это означает, что коэффициенты теплового ядра будут тоже совпадать. Регуляризованная энергия в случае трехмерного пространства для граничных условий ТЕ- и ТМ1-типов имеет следующий вид:

$$
\begin{gathered}
E_{\lambda}^{\mathrm{TE}}(s)=\frac{\mu^{2 s} \cos (\pi s)}{8 \pi^{2}(s-3 / 2)} \int_{0}^{\infty} d k k^{3-2 s} \frac{k L \lambda \operatorname{sech}^{2}(k L)-\lambda \operatorname{th}(k L)}{2 k^{2}+\lambda \operatorname{th}(k L) k} \\
E_{\lambda}^{\mathrm{TM} 1}(s)=\frac{\mu^{2 s} \cos (\pi s)}{8 \pi^{2}(s-3 / 2)} \int_{0}^{\infty} d k k^{3-2 s} \frac{-k L \lambda \operatorname{csch}^{2}(k L)-\lambda \operatorname{cth}(k L)}{2 k^{2}+\lambda \operatorname{cth}(k L) k} .
\end{gathered}
$$

При $s \rightarrow 0$ интегралы расходятся, как $k^{2}$. Для получения конечного результата мы должны выделить в явном виде все расходимости выражений (23). По этой причине мы вычитаем и добавляем к подынтегральному выражению первые три члена его разложения по степеням $z=1 /(k+\lambda / 2)$ и затем переходим к пределу $s \rightarrow 0$. Регуляризованная энергия при этом разбивается на две части: рассчитываемую численно $E_{\lambda}^{\text {num }}$ и аналитическую $E_{\lambda}^{\text {an }}$, которая совпадает для ТЕ- и ТМ1-случаев: $E_{\lambda}(s)=E_{\lambda}^{\mathrm{num}}+E_{\lambda}^{\mathrm{an}}(s)$, где

$$
\begin{aligned}
E_{\lambda, \mathrm{TE}}^{\mathrm{num}} & =-\frac{1}{12 \pi^{2}} \int_{0}^{\infty} d k k^{3}\left\{\frac{k L \lambda \operatorname{sech}^{2}(k L)-\lambda \operatorname{th}(k L)}{2 k^{2}+\lambda \operatorname{th}(k L) k}+\frac{\lambda}{2 z^{2}}+\frac{\lambda^{2}}{4 z^{3}}+\frac{\lambda^{3}}{8 z^{4}}\right\}= \\
& =\frac{\lambda^{3}}{32 \pi^{2}} \int_{0}^{\infty} d x x^{2}\left\{\ln \left(1+\frac{\operatorname{th}(x p)}{x}\right)-\frac{1}{x+1}-\frac{1}{2(x+1)^{2}}-\frac{1}{3(x+1)^{3}}\right\}, \\
E_{\lambda, \mathrm{TM} 1}^{\mathrm{num}} & =\frac{\lambda^{3}}{32 \pi^{2}} \int_{0}^{\infty} d x x^{2}\left\{\ln \left(1+\frac{\operatorname{cth}(x p)}{x}\right)-\frac{1}{x+1}-\frac{1}{2(x+1)^{2}}-\frac{1}{3(x+1)^{3}}\right\}, \\
E_{\lambda}^{\mathrm{an}}(s) & =-\frac{\mu^{2 s} \cos (\pi s)}{8 \pi^{2}(s-3 / 2)} \int_{0}^{\infty} d k k^{3-2 s}\left\{\frac{\lambda}{2 z^{2}}+\frac{\lambda^{2}}{4 z^{3}}+\frac{\lambda^{3}}{8 z^{4}}\right\}=\frac{\mu^{2 s}}{24 \pi}\left(\frac{\lambda}{2}\right)^{3-2 s} \frac{1+2 s^{2}}{\sin (\pi s)}= \\
& =\frac{a_{2}}{16 \pi^{3 / 2}}\left\{\frac{\Gamma(s)}{\Gamma(s-1 / 2)}+\frac{2 \ln (\mu / \lambda)}{\Gamma(s-1 / 2)}\right\}+\frac{\lambda^{3}}{96 \pi^{2}}+O(s) ;
\end{aligned}
$$

здесь $p=L \lambda / 2$ и $a_{2}=-\lambda^{3} / 6$.

Проанализируем регуляризованные выражения для энергий в двух предельных случаях $\lambda \rightarrow \infty$ и $\lambda \rightarrow 0$, что соответствует пределам $p \rightarrow \infty$ и $p \rightarrow 0$. Ожидается, что в этих пределах энергия вакуумных флуктуаций для скалярного поля при наличии граничных условий TE- и TM1-типов будет равна стандартной энергии Казимира для двух идеально проводящих пластин без сингулярного источника. 
В пределе $\lambda \rightarrow \infty$ получим

$$
\begin{aligned}
E_{\lambda}^{\mathrm{TE}}(s) & =\frac{a_{2}}{16 \pi^{3 / 2}} \frac{\Gamma(s)+2 \ln (\mu / \lambda)}{\Gamma(s-1 / 2)}+\frac{\lambda^{3}}{72 \pi^{2}}-\frac{\pi^{2}}{768 L^{3}}+O(s), \\
E_{\lambda}^{\mathrm{TM} 1}(s) & =\frac{a_{2}}{16 \pi^{3 / 2}} \frac{\Gamma(s)+2 \ln (\mu / \lambda)}{\Gamma(s-1 / 2)}+\frac{\lambda^{3}}{72 \pi^{2}}+\frac{\pi^{2}}{768 L^{3}}+O(s) .
\end{aligned}
$$

Для перенормировки мы вычитаем все слагаемые, которые отличны от нуля в пределе $\lambda \rightarrow \infty$. Таким образом, полная перенормированная энергия в пределе $\lambda \rightarrow \infty$ принимает следующие значения:

$$
E_{\mathrm{TE}}=-\frac{\pi^{2}}{1440 L^{3}}-\frac{\pi^{2}}{1440 L^{3}}, \quad E_{\mathrm{TM} 1}=\frac{7}{8} \frac{\pi^{2}}{1440 L^{3}}+\frac{7}{8} \frac{\pi^{2}}{1440 L^{3}} .
$$

Как и ожидалось, полученные выражения представляют собой сумму значений стандартных энергий Казимира для двух пластин, удаленных на расстояние $L$ друг от друга.

Выделим расходящуюся часть энергии

$$
E^{\operatorname{div}}(s)=\frac{a_{2}}{16 \pi^{3 / 2}} \frac{\Gamma(s)+2 \ln (\mu / \lambda)}{\Gamma(s-1 / 2)}+\frac{\lambda^{3}}{72 \pi^{2}},
$$

в результате получаем следующие перенормированные выражения для энергии:

$$
E_{\mathrm{TE}}=-\frac{\pi^{2}}{1440(2 L)^{3}}+E_{\lambda, \mathrm{TE}}, \quad E_{\mathrm{TM} 1}=-\frac{\pi^{2}}{1440(2 L)^{3}}+E_{\lambda, \mathrm{TM} 1},
$$

где

$$
\begin{aligned}
E_{\lambda, \mathrm{TE}} & =\frac{\lambda^{3}}{32 \pi^{2}} \int_{0}^{\infty} d x x^{2}\left\{\ln \left(1+\frac{\operatorname{th}(x p)}{x}\right)-\frac{1}{y}-\frac{1}{2 y^{2}}-\frac{1}{3 y^{3}}\right\}-\frac{\lambda^{3}}{288 \pi^{2}}, \\
E_{\lambda, \mathrm{TM} 1} & =\frac{\lambda^{3}}{32 \pi^{2}} \int_{0}^{\infty} d x x^{2}\left\{\ln \left(1+\frac{\operatorname{cth}(x p)}{x}\right)-\frac{1}{y}-\frac{1}{2 y^{2}}-\frac{1}{3 y^{3}}\right\}-\frac{\lambda^{3}}{288 \pi^{2}} .
\end{aligned}
$$

В пределе $\lambda \rightarrow 0$

$$
E_{\lambda}=\mp \frac{\lambda}{184 L^{2}} \rightarrow 0
$$

и полная энергия совпадает со стандартной энергией Казимира для двух пластин, удаленных на расстояние $2 L$ друг от друга.

Перенормированная энергия нулевых колебаний имеет следующий вид: для фиксированного $\lambda$

$$
\frac{E_{\mathrm{TE}}}{\lambda^{3}}=\frac{1}{32 \pi^{2}} \int_{0}^{\infty} d x x^{2}\left\{\ln \left(1+\frac{\operatorname{th}(x p)}{x}\right)-\frac{1}{y}-\frac{1}{2 y^{2}}-\frac{1}{3 y^{3}}-\frac{1}{3 y^{4}}\right\}-\frac{\pi^{2}}{1440(4 p)^{3}},
$$

для фиксированного $L$

$$
E_{\mathrm{TE}} \cdot(2 L)^{3}=\frac{2 p^{3}}{\pi^{2}} \int_{0}^{\infty} d x x^{2}\left\{\ln \left(1+\frac{\operatorname{th}(x p)}{x}\right)-\frac{1}{y}-\frac{1}{2 y^{2}}-\frac{1}{3 y^{3}}-\frac{1}{3 y^{4}}\right\}-\frac{\pi^{2}}{1440} .
$$




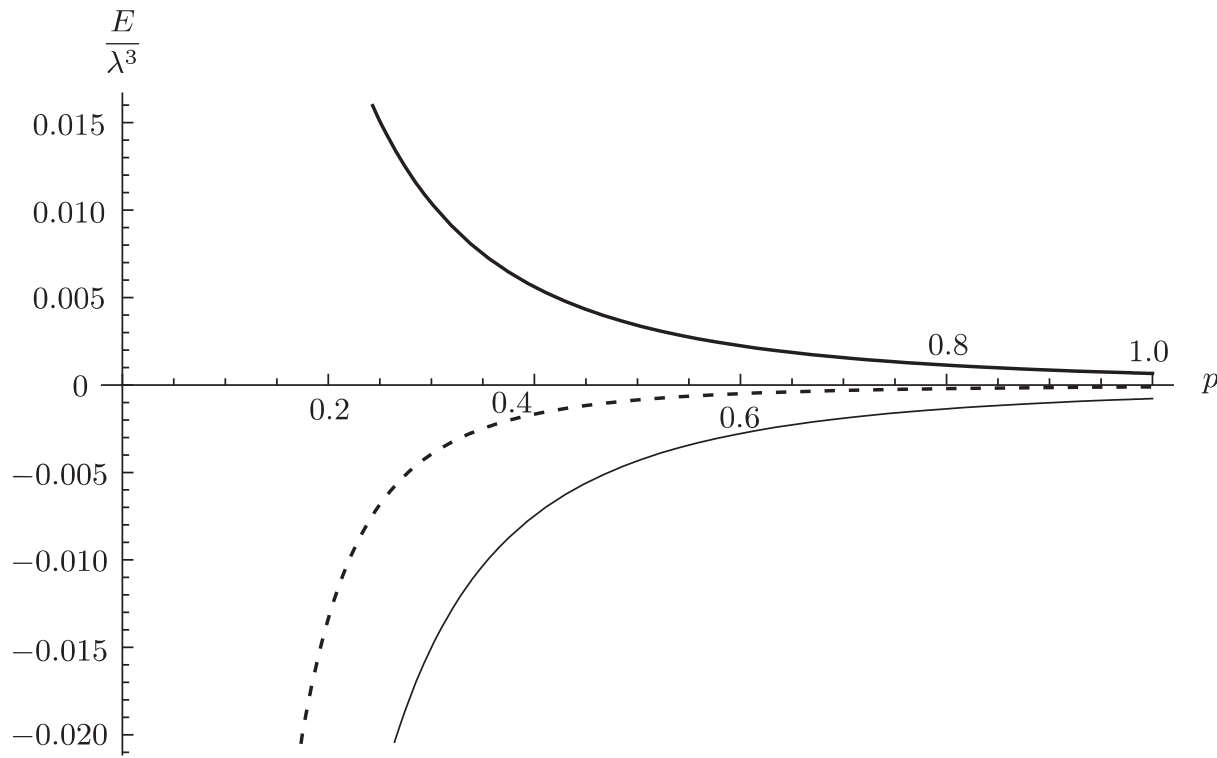

Рис. 1. Зависимость энергии нулевых колебаний для двух пластин, находящихся на расстоянии $L$, от $p=L \lambda / 2$ при фиксированном $\lambda$. Обычная энергия Казимира между двумя пластинами, удаленными на расстояние $L$ друг от друга, представлена штриховой линией. График энергии в ТМ1-случае находится выше оси $p$ (жирная линия), а в ТЕ-случае - ниже оси $p$ (тонкая линия).
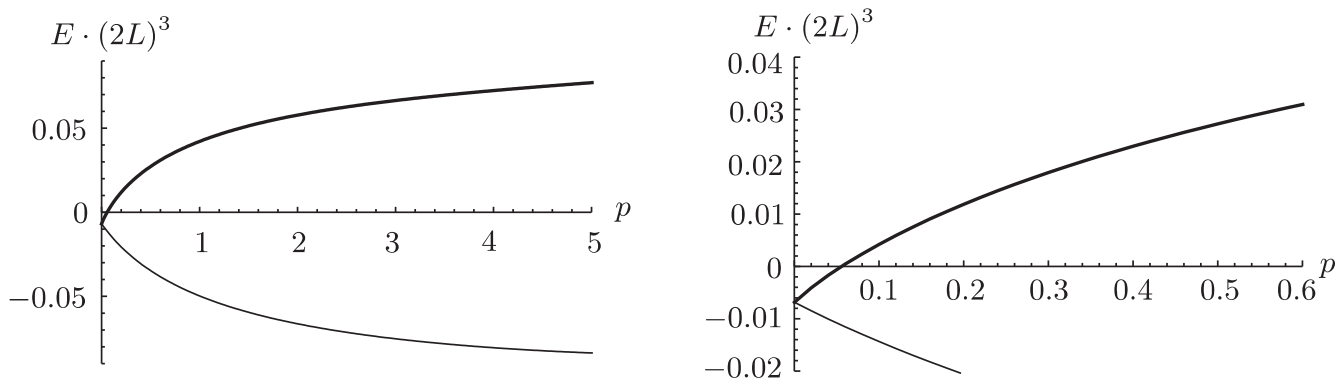

Рис. 2. Зависимость энергии нулевых колебаний от $\lambda$ при фиксированном $L$ для ТМ1-случая (жирная линия) и ТЕ-случая (тонкая линия). На правом рисунке те же кривые в окрестности начала координат.

$\mathrm{C}$ помощью замены $\operatorname{th}(x p) \mapsto \operatorname{cth}(x p)$ получаем выражения для граничных условий ТМ1-типа.

Результаты численного анализа энергии нулевых колебаний при фиксированных $\lambda$ и $L$ представлены на рис. 1 и 2. Из рис. 1 видно, что при бесконечном удалении границ (при $L \rightarrow \infty$ ) энергия нулевых колебаний и стандартная энергия Казимира стремятся к нулю, как и должно быть, поскольку энергия Казимира в 
пустом пространстве равна нулю. Из рис. 2 видно, что для предельных значений $\lambda \rightarrow 0$ и $\lambda \rightarrow \infty$ энергия нулевых колебаний принимает значения, согласующиеся с теорией. При $\lambda \rightarrow 0$ энергия равна $(2 L)^{3} E_{\mathrm{Cas}}=-\pi^{2} / 1440$ и переходит в стандартную энергию Казимира для двух пластин, удаленных на расстояние $2 L$ друг от друга. При $\lambda \rightarrow \infty$ энергия равна $16 \pi^{2} / 1440$ и одинакова, с точностью до знака, для граничных условий ТM1- и ТЕ-типов. Отметим, что в случае граничных условий TM1-типа энергия нулевых колебаний принимает нулевое значение при определенном значении параметра $p=\lambda L / 2$, что соответствует $\lambda L \approx 0.11$ (см. рис. 2 ).

4.2. Массивное скалярное поле. Рассмотрим эту же задачу в случае массивного поля. Выделим расходимость при $s \rightarrow 0$ :

$$
\begin{aligned}
E_{\lambda}^{\mathrm{an}}(s)= & -\frac{\mu^{2 s} \cos (\pi s)}{8 \pi^{2}(s-3 / 2)} \int_{m}^{\infty} d k\left(k^{2}-m^{2}\right)^{3 / 2-s}\left\{\frac{\lambda}{2 z^{2}}+\frac{\lambda^{2}}{4 z^{3}}+\frac{\lambda^{3}}{8 z^{4}}\right\}= \\
= & -\frac{a_{2}}{32 \pi^{2}}\left(\frac{1}{s}+2 \ln \frac{\mu}{m}\right)-\frac{\lambda}{96 \pi^{2}\left(4 m^{2}-\lambda^{2}\right)}\left\{-12(1-2 \ln 2) m^{4}+\right. \\
& +\lambda^{2}(1-10 \ln 2) m^{2}+\lambda^{4} \ln 2- \\
& \left.-\frac{\lambda\left(24 m^{4}-12 \lambda^{2} m^{2}+\lambda^{4}\right) \arccos (\lambda / 2 m)}{\sqrt{4 m^{2}-\lambda^{2}}}\right\}+O(s),
\end{aligned}
$$

где $a_{2}=-\lambda^{3} / 6+m^{2} \lambda$. Конечная часть энергии рассчитывается численно:

$$
\begin{aligned}
E_{\lambda, \mathrm{TE}}^{\mathrm{num}}= & \frac{\lambda^{3}}{32 \pi^{2}} \int_{q}^{\infty} d x x \sqrt{x^{2}-q^{2}} \times \\
& \times\left\{\ln \left(1+\frac{\operatorname{th}(x p)}{x}\right)-\frac{1}{x+1}-\frac{1}{2(x+1)^{2}}-\frac{1}{3(x+1)^{3}}\right\}, \\
E_{\lambda, \mathrm{TM} 1}^{\mathrm{num}}= & \frac{\lambda^{3}}{32 \pi^{2}} \int_{q}^{\infty} d x x \sqrt{x^{2}-q^{2}} \times \\
& \times\left\{\ln \left(1+\frac{\operatorname{cth}(x p)}{x}\right)-\frac{1}{x+1}-\frac{1}{2(x+1)^{2}}-\frac{1}{3(x+1)^{3}}\right\},
\end{aligned}
$$

где $q=2 m / \lambda$. Следовательно, мы имеем два параметра $p$ и $q$ и можем сравнивать $1 / \lambda$ с расстоянием $L$ между пластинами и комптоновской длиной волны частиц $1 / m$. Чтобы получить конечное выражение для энергии нулевых колебаний, рассмотрим различные способы перенормировок, в том числе стандартный.

Рассмотрим сначала подход, применяемый в случае безмассового скалярного поля. Выделим все слагаемые, которые сохраняются в пределе $\lambda \rightarrow \infty$ :

$$
E_{\mathrm{TE}}^{\mathrm{div}}(s)=-\frac{a_{2}}{32 \pi^{2}}\left(\frac{1}{s}+2 \ln \frac{2 \mu}{\lambda}\right)+\frac{\lambda^{3}}{9 \cdot 32 \pi^{2}} ;
$$

вычитая (26) из (25), получаем перенормированное выражение для энергии нулевых колебаний:

$$
\begin{aligned}
E_{\mathrm{TE}}= & \frac{\lambda^{3}}{32 \pi^{2}} J(p, q)-\frac{\lambda^{3}}{9 \cdot 32 \pi^{2}}-\frac{\lambda}{96 \pi^{2}\left(4 m^{2}-\lambda^{2}\right)}\left\{-12(1-2 \ln 2) m^{4}+\right. \\
& \left.+\lambda^{2}(1-10 \ln 2) m^{2}+\lambda^{4} \ln 2-\frac{\lambda\left(24 m^{4}-12 \lambda^{2} m^{2}+\lambda^{4}\right) \arccos (\lambda / 2 m)}{\sqrt{4 m^{2}-\lambda^{2}}}\right\}
\end{aligned}
$$


где

$$
\begin{aligned}
J(p, q)= & \frac{\beta^{3}}{p^{3}} \int_{1}^{\infty} d y y \sqrt{y^{2}-1} \times \\
& \times\left\{\ln \left(1+\frac{p \operatorname{th}(\beta y)}{\beta y}\right)-\frac{1}{1+y \beta / p}-\frac{1}{2(1+y \beta / p)^{2}}-\frac{1}{3(1+y \beta / p)^{3}}\right\} .
\end{aligned}
$$

В пределе $m \rightarrow \infty$ выражение (27) сохраняет расходимость вида $\lambda m^{2}(1-2 \ln 2) / 32 \pi^{2}$. Следовательно, данный способ перенормировки является неудовлетворительным.

Далее, рассмотрим стандартный способ перенормировки - выделение всех слагаемых, сохраняющихся в пределе $m \rightarrow \infty$. В этом пределе расходящаяся часть энергии выглядит следующим образом:

$$
E_{\mathrm{TE}}^{\mathrm{div}}(s)=-\frac{a_{2}}{32 \pi^{2}}\left(\frac{1}{s}+2 \ln \frac{\mu}{m}\right)+\frac{(-1+\ln 2) \lambda^{3}}{96 \pi^{2}}+\frac{m \lambda^{2}}{64 \pi}+\frac{m^{2}(1-2 \ln 2) \lambda}{32 \pi^{2}} .
$$

Вычитая (28) из (25), получаем выражение для перенормированной энергии:

$$
\begin{aligned}
E_{\mathrm{TE}}= & \frac{\lambda^{3}}{32 \pi^{2}} J(p, q)-\frac{\lambda}{96 \pi^{2}\left(4 m^{2}-\lambda^{2}\right)}\left\{-12(1-2 \ln 2) m^{4}+\lambda^{2}(1-10 \ln 2) m^{2}+\right. \\
& \left.+\lambda^{4} \ln 2-\frac{\lambda\left(24 m^{4}-12 \lambda^{2} m^{2}+\lambda^{4}\right) \arccos (\lambda / 2 m)}{\sqrt{4 m^{2}-\lambda^{2}}}\right\}- \\
& -\frac{(-1+\ln 2) \lambda^{3}}{96 \pi^{2}}-\frac{m \lambda^{2}}{64 \pi}-\frac{m^{2}(1-2 \ln 2) \lambda}{32 \pi^{2}} .
\end{aligned}
$$

В пределе $\lambda \rightarrow \infty$ сохраняется логарифмическая расходимость:

$$
E_{\mathrm{TE}}=\frac{(\ln (m / \lambda)+1) \lambda^{3}}{96 \pi^{2}}-\frac{m \lambda^{2}}{64 \pi}-\frac{m^{2}(6 \ln (m / \lambda)+1) \lambda}{96 \pi^{2}} .
$$

Таким образом, этот способ перенормировки не удовлетворяет предельным случаям.

Рассмотрим, наконец, перенормировку, при которой необходимо выделить слагаемые, сохраняющиеся в пределе $L \rightarrow \infty$ :

$$
\begin{aligned}
E_{\mathrm{TE}}^{\mathrm{div}}(s)= & \frac{\lambda^{3}}{32 \pi^{2}} J(\infty, q)-\frac{a_{2}}{32 \pi^{2}}\left(\frac{1}{s}+2 \ln \frac{\mu}{m}\right)- \\
& -\frac{\lambda}{96 \pi^{2}\left(4 m^{2}-\lambda^{2}\right)}\left\{-12(1-2 \ln 2) m^{4}+\lambda^{2}(1-10 \ln 2) m^{2}+\lambda^{4} \ln 2-\right. \\
& \left.-\frac{\lambda\left(24 m^{4}-12 \lambda^{2} m^{2}+\lambda^{4}\right) \arccos (\lambda / 2 m)}{\sqrt{4 m^{2}-\lambda^{2}}}\right\} .
\end{aligned}
$$

Вычитая (29) из (25), получим выражение для перенормированной энергии:

$$
E_{\mathrm{TE}}=\frac{\lambda^{3}}{32 \pi^{2}} J(p, q)-\frac{\lambda^{3}}{32 \pi^{2}} J(\infty, q)+E_{\mathrm{Cas}}^{\mathrm{DD}}(2 L) .
$$

В пределе $m \rightarrow \infty$ имеем $E_{\mathrm{TE}}=O\left(m^{-2}\right)$. В пределе $\lambda \rightarrow \infty$ получаем

$$
E_{\mathrm{TE}}=2 E_{\mathrm{Cas}}^{\mathrm{TE}}(L)-E_{\mathrm{Cas}}^{\mathrm{TE}}(2 L)+E_{\mathrm{Cas}}^{\mathrm{TE}}(2 L)=2 E_{\mathrm{Cas}}^{\mathrm{TE}}(L) .
$$



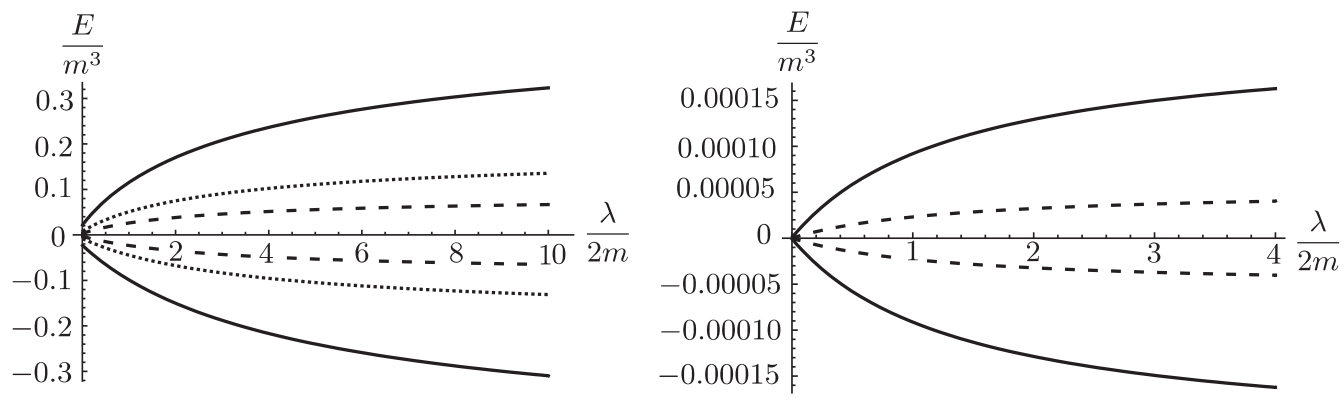

Рис. 3. Зависимость энергии от $\lambda / 2 m$ при фиксированном параметре $\beta=m L$ для $\beta=0.3$ (сплошная линия), $\beta=0.4$ (пунктир) и $\beta=0.5$ (штриховая линия); графики для случая ТМ1-поляризации расположены выше оси абсцисс, для случая ТЕ-поляризации - ниже оси абсцисс. На правом рисунке аналогичные кривые для $\beta=2$ (сплошная линия) и $\beta=2.5$ (штриховая линия). Легко видеть, что при увеличении $\beta$ энергия нулевых колебаний для любого $\lambda$ стремится к нулю.

Таким образом, мы имеем конечное выражение, и данный способ перенормировки является корректным. Окончательные выражения для перенормированной энергии в TE- и TM1-случаях будут выглядеть следующим образом:

$$
\begin{aligned}
E_{\mathrm{TE}} & =\frac{\lambda^{3}}{32 \pi^{2}} \int_{q}^{\infty} d x x \sqrt{x^{2}-q^{2}} \ln \left(\frac{x+\operatorname{th}(x p)}{x+1}\right)+E_{\mathrm{CaS}}^{\mathrm{DD}}(2 L), \\
E_{\mathrm{TM} 1} & =\frac{\lambda^{3}}{32 \pi^{2}} \int_{q}^{\infty} d x x \sqrt{x^{2}-q^{2}} \ln \left(\frac{x+\operatorname{cth}(x p)}{x+1}\right)+E_{\mathrm{Cas}}^{\mathrm{DN}}(2 L) .
\end{aligned}
$$

Результаты численного анализа энергии для случаев ТМ- и ТЕ-поляризаций представлены на рис. 3. Графики демонстрируют поведение энергии нулевых колебаний массивного скалярного поля для произвольного $\lambda$. При $\lambda \rightarrow 0$ энергия вакуумных флуктуаций должна принимать постоянное значение, зависящее от параметра $\beta$, что хорошо видно на левом рисунке. Энергия вакуумных флуктуаций при $\lambda=0$ равна стандартной энергии Казимира для двух пластин, удаленных на расстояние $2 L$ друг от друга, при определенном значении параметра $\beta$. Отметим, что при $\beta \rightarrow \infty$ (что соответствует $m \rightarrow \infty$ ) энергия нулевых колебаний, как и любая другая энергия, включая стандартную энергию Казимира, должна принимать нулевое значение при любых $\lambda$. Это хорошо видно на правой части рис. 3 , где уже для $\beta=2$ и $\beta=2.5$ энергия принимает практически нулевое значение для любых $\lambda$.

\section{5. ЗАКЛЮЧЕНИЕ}

На основе метода дзета-регуляризации вычислена энергия вакуумных флуктуаций скалярного поля при нетривиальных граничных условиях, аналогичных условиям для ТЕ- и ТМ-поляризаций электромагнитного поля при наличии проводящей бесконечно тонкой сферы. Параметр $\lambda$ имеет смысл волнового вектора плазмона, "живущего" на этой сфере. Для массивного и безмассового полей получены соответствующие дзета-функции и вычислены все коэффициенты теплового ядра. 
Рассмотренные граничные условия соответствуют некоторым сингулярным потенциалам. В пределе $\lambda \rightarrow \infty$ граничные условия ТЕ- и ТМ-типов переходят в граничные условия Дирихле и Неймана соответственно. С другой стороны, применение стандартной схемы вычитания слагаемых, выживающих в пределе больших масс, приводит к неудовлетворительному выражению, поскольку в пределе $\lambda \rightarrow \infty$ полученное выражение расходится.

В случае безмассового поля в задаче имеются два размерных параметра $\lambda$ и $L$, которые входят в выражения для энергии в безразмерной комбинации $p=\lambda L / 2$. По этой причине слагаемые, выживающие в пределе Дирихле $\lambda \rightarrow \infty$, такие же, как и в пределе $L \rightarrow \infty$, и поэтому полученные выражения для перенормированной энергии принимают физически разумные значения в пределах $\lambda \rightarrow 0$ и $\lambda \rightarrow \infty$. В первом пределе мы имеем выражение, соответствующее наличию двух пластин с граничными условиями Дирихле на расстоянии $2 L$ друг от друга, во втором случае нулевое значение энергии Казимира, как и должно быть для уединенной пластины.

При наличии массивного поля имеются три размерных параметра $\lambda, L$ и $m$, которые входят в формулы в виде двух безразмерных комбинаций $q=2 m / \lambda$ и $p=\lambda L / 2$. Вычитание слагаемых, выживающих в пределах $\lambda \rightarrow \infty$ или $m \rightarrow \infty$, приводит к неудовлетворительным результатам. В первом случае (при $\lambda \rightarrow \infty$ ) полученное выражение расходится в пределе $m \rightarrow \infty$, во втором случае (при $m \rightarrow \infty$ ), наоборот, расходится в пределе $\lambda \rightarrow \infty$. Вычитание слагаемых, выживающих в пределе $L \rightarrow \infty$, приводит к удовлетворительному во всех отношениях выражению для перенормированной энергии. В пределе $\lambda \rightarrow \infty$ получаем энергии Казимира для трех пластин, т.е. сингулярный потенциал в этом пределе приводит к появлению пластины с условием Дирихле (в ТЕ-случае) или Неймана (в ТМ-случае) в месте нахождения потенциала. В пределе $m \rightarrow \infty$ энергия равна нулю, поскольку в классическом пределе флуктуации отсутствуют. Наконец, в пределе $\lambda \rightarrow 0$ получаем энергию Казимира для двух пластин, находящихся на расстоянии $2 L$.

\section{ПРИЛОЖЕНИЕ}

Коэффициенты теплового ядра для $N$-мерного пространства можно найти, используя следующую формулу:

$$
a_{n / 2}=(4 \pi)^{N / 2} \operatorname{Res}_{(N-n) / 2}\left(\Gamma(s) \zeta\left(s, D_{N}\right)\right), \quad n=0,1,2, \ldots .
$$

Это представление удобно как в массивном, так и в безмассовом случаях. Для массивного случая коэффициенты теплового ядра, полученные при разложении по степеням массы, будем обозначать через $b_{k}$. Используя выражение, связывающее дзета-функцию и след теплового ядра $K(t)$,

$$
\zeta\left(s, D_{N}\right)=\int_{0}^{\infty} d t \frac{t^{s-1}}{\Gamma(s)} K(t),
$$

получаем асимптотическое разложение для дзета-функции. След теплового ядра имеет следующее асимптотическое разложение по степеням $t$ :

$$
K_{\mathrm{as}}(t)=\frac{e^{-t m^{2}}}{(4 \pi t)^{N / 2}} \sum_{n=0}^{\infty} b_{n / 2} t^{n / 2} .
$$


Учитывая (32) и (33), получим

$$
\zeta_{\text {as }}\left(s, D_{N}\right)=\frac{1}{(4 \pi)^{N / 2}} \sum_{n=0}^{\infty} b_{n / 2} m^{-2 s-n+d} \frac{\Gamma(s+(n-N) / 2)}{\Gamma(s)} .
$$

Подставляя это разложение дзета-функции в (31), имеем

$$
a_{j}=\sum_{n=0}^{j} b_{n} \frac{\left(-m^{2}\right)^{j-n}}{(j-n) !}, \quad a_{j+1 / 2}=\sum_{n=0}^{j} b_{n+1 / 2} \frac{\left(-m^{2}\right)^{j-n}}{(j-n) !}
$$

где коэффициенты $b_{k}$ теплового ядра для массивного случая определяются выражением (33), а коэффициенты $a_{k}$ - выражением (31).

Применительно к нашей ситуации в случае $m=0$ мы имеем следующие коэффициенты теплового ядра:

$$
\begin{aligned}
a_{0} & =-\frac{2}{\lambda}, & a_{1 / 2} & =\sqrt{\pi} \\
a_{n} & =-\left(\frac{\lambda}{2}\right)^{2 n-1} \frac{\sqrt{\pi}}{\Gamma(n+1 / 2)}, & a_{n+1 / 2} & =\left(\frac{\lambda}{2}\right)^{2 n} \frac{\sqrt{\pi}}{n !} .
\end{aligned}
$$

В случае массивного поля для дзета-функции (20), используя (31), получаем

$$
\begin{aligned}
a_{0} & =0, \quad a_{1 / 2}=0, \quad a_{1}=-\lambda=b_{1}, \quad a_{3 / 2}=\frac{\sqrt{\pi}}{4} \lambda^{2}=b_{3 / 2}, \\
a_{2} & =-\frac{\lambda^{3}}{6}+m^{2} \lambda=b_{2}-m^{2} b_{1}, \quad a_{5 / 2}=\frac{\sqrt{\pi}}{32}\left(\lambda^{4}-8 m^{2} \lambda^{2}\right)=b_{5 / 2}-m^{2} b_{3 / 2} \\
a_{3} & =-\frac{\lambda^{5}}{60}+\frac{m^{2} \lambda^{3}}{6}-\frac{m^{4} \lambda}{2}=b_{3}-m^{2} b_{2}+\frac{1}{2} m^{4} b_{1}, \\
a_{7 / 2} & =\frac{\sqrt{\pi}}{384}\left(\lambda^{6}-12 m^{2} \lambda^{2}+48 m^{4}\right)=b_{7 / 2}-m^{2} b_{5 / 2}+\frac{1}{2} m^{4} b_{3 / 2} .
\end{aligned}
$$

Для нахождения коэффициентов теплового ядра $b_{k}$ используем формулу (19) и разложение по степеням $1 / k$ :

$$
\begin{aligned}
\zeta_{\lambda}\left(s, D_{1}\right) & =-\frac{\sin (\pi s)}{\pi} \int_{m}^{\infty} d k\left(k^{2}-m^{2}\right)^{-s} \frac{\lambda}{2 k^{2}} \sum_{n=0}^{\infty}\left(-\frac{\lambda}{2 k}\right)^{n}= \\
& =\frac{\sin (\pi s)}{\pi} \sum_{n=0}^{\infty}\left(-\frac{\lambda}{2}\right)^{n+1} m^{-2 s-n-1} \int_{1}^{\infty} d x\left(x^{2}-1\right)^{-s} x^{-n-2} .
\end{aligned}
$$

Принимая во внимание равенство

$$
\int_{1}^{\infty} d x\left(x^{2}-1\right)^{-s} x^{-n-2}=\frac{\pi}{\sin (\pi s) \Gamma(s)} \frac{\Gamma(s+(1+n) / 2)}{2 \Gamma((3+n) / 2)},
$$

получаем выражение для дзета-функции одномерного оператора Лапласа:

$$
\zeta_{\lambda}\left(s, D_{1}\right)=\sum_{n=2}^{\infty}\left(-\frac{\lambda}{2}\right)^{n-1} m^{-2 s-n+1} \frac{1}{\Gamma(s)} \frac{\Gamma(s+(n-1) / 2)}{2 \Gamma((n+1) / 2)} .
$$


Сравнивая (38) и (34), получим коэффициенты теплового ядра:

$$
b_{0}=0, \quad b_{1 / 2}=0, \quad b_{n / 2}=\left(-\frac{\lambda}{2}\right)^{n-1} \frac{\sqrt{\pi}}{\Gamma((n+1) / 2)} .
$$

Благодарности. Работа авторов была частично финансирована РФФИ (грант № 08-02-00325_а).

\section{Список литературы}

[1] H. B. G. Casimir, Phys. Rev., 51 (1948), 793-795.

[2] M. Bordag, G. L. Klimchitskaya, U. Mohideen, V.M. Mostepanenko, Advances in the Casimir Effect, Internat. Ser. Monogr. Phys., 145, Oxford Univ. Press, USA, 2009.

[3] M. Bordag, Eighth Workshop on Quantum Field Theory under the Influence of External Conditions, IOP, New York, 2008.

[4] Ф. А. Березин, Л. Д. Фаддеев, Докл. АН СССР, 137 (1961), 1011-1014.

[5] М. Рид, Б. Саймон, Методы современной математической физики, т. 2: Гармонический анализ. Самосопряженность, Мир, М., 1978.

[6] С. Альбеверио, Ф. Гестези, Р. Хеэг-Крон, Х. Холден, Решаемые модели в квантовой механике, Мир, М., 1991.

[7] С. Г. Мамаев, Н. Н. Трунов, ЯФ, 35 (1982), 1049-1058.

[8] M. Bordag, D. Henning, D. Robaschik, J. Phys. A, 25:16 (1992), 4483-4498.

[9] M. Scandurra, J. Phys. A, 32:30 (1999), 5679-5691, arXiv: hep-th/9811164.

[10] M. Bordag, D. V. Vassilevic, J. Phys. A, 32:47 (1999), 8247-8259, arXiv: hep-th/9907076.

[11] M. Bordag, K. Kirsten, D. Vassilevich, Phys. Rev. D, 59:8 (1999), 085011, 14 pp., arXiv: hep-th/9811015.

[12] M. Scandurra, J. Phys. A, 33:32 (2000), 5707-5718, arXiv: hep-th/0004051.

[13] G. Barton, J. Phys. A, 37:3 (2004), 1011-1049.

[14] K. A. Milton, J. Phys. A, 37:24 (2004), 6391-6406, arXiv: hep-th/0401090.

[15] M. Bordag, I. G. Pirozhenko, V. V. Nesterenko, J. Phys. A, 38:50 (2005), 11027-11043, arXiv: hep-th/0508198.

[16] M. Bordag, J. Phys. A, 39:21 (2006), 6173 - 6185, arXiv: hep-th/0511269.

[17] N. R. Khusnutdinov, Phys. Rev. D, 73:2 (2006), 025003, 13 pp., arXiv: hep-th/0511038.

[18] M. Bordag, Phys. Rev. D, 76:6 (2007), 065011, 14 pp., arXiv: 0704.3845.

[19] С. Г. Трунов, Н. Н. Мамаев, Изв. вузов. Сер. физ., 7 (1980), 9-13.

[20] R. L. Jaffe, L. R. Williamson, Ann. Phys., 282:2 (2000), 432-448, arXiv: hep-th/9907199.

[21] N. Graham, R. L. Jaffe, V. Khemani, M. Quandt, O. Schröder, H. Weigel, Nucl. Phys. B, 677:1-2 (2004), 379-404, arXiv: hep-th/0309130.

[22] R. L. Jaffe, Phys. Rev. D, 72:2 (2005), 021301, 5 pp., arXiv: hep-th/0503158.

[23] M. P. Hertzberg, R. L. Jaffe, M. Kardar, A. Scardicchio, Phys. Rev. D, 76:4 (2007), 045016, 13 pp., arXiv: 0705.0139.

[24] T. Emig, N. Graham, R. L. Jaffe, M. Kardar, Phys. Rev. D, 77:2 (2008), 025005, 23 pp., arXiv: 0710.3084.

[25] J. S. Dowker, R. Critchley, Phys. Rev. D, 13:12 (1976), 3224-3232.

[26] S. W. Hawking, Comm. Math. Phys., 55:2 (1977), 133-148.

[27] D. V. Vassilevich, Phys. Rep., 388:5-6 (2003), 279-360, arXiv: hep-th/0306138.

[28] S. K. Blau, M. Visser, A. Wipf, Nucl. Phys. B, 310:1 (1988), 163-180, arXiv: 0906.2817.

[29] M. Bordag, K. Kirsten, Phys. Rev. D, 53:10 (1996), 5753-5760, arXiv: hep-th/9608070.

[30] M. Bordag, E. Elizalde, K. Kirsten, S. Leseduarte, Phys. Rev. D, 56:8 (1997), 4896-4904, arXiv: hep-th/9608071. 
[31] M. Bordag, K. Kirsten, D. Vassilevich, Phys. Rev. D, 59:8 (1999), 085011, 14 pp., arXiv: hep-th/9811015.

[32] S. Minakshisundaram, Å. Pleijel, Canadian J. Phys., 1 (1949), 242-256.

[33] S. Minakshisundaram, J. Indian Math. Soc., 17 (1954), 159-165.

[34] M. Bordag, I. G. Pirozhenko, V. V. Nesterenko, J. Phys. A, 38:50 (2005), 11027-11043, arXiv: hep-th/0508198.

[35] M. Bordag, I. V. Fialkovsky, D. M. Gitman, D. V. Vassilevich, Phys. Rev. B, 80:24 (2009), 245406, 5 pp., arXiv: 0907.3242.

[36] D. V. Vassilevich, Phys. Rev. D, 79:6 (2009), 065016, 11 pp., arXiv: 0901.0337.

Поступила в редакцию 26.05.2010, после доработки 8.06.2010 